\title{
Sistema de Competição Lotka-Volterra Sob Ruído Branco
}

\author{
David Zavaleta Villanueva \\ Depto. de Matemática, CCET, UFRN, \\ Campus Lagoa Nova \\ 59078-970, Natal, UFRN \\ E-mail: villanueva@ccet.ufrn.br
}

Resumo: A consideração do ruído branco multiplicativo muda drasticamente o comportamento do sistema de Lotka-Volterra

$$
\left\{\begin{array}{l}
\dot{u}=u\left(a_{1}-b_{1} u-c_{1} v\right) \\
\dot{v}=v\left(a_{2}-b_{2} u-c_{2} v\right)
\end{array}\right.
$$

e apresenta comportamentos que não tem análogos no caso determinístico. A evolução no tempo da competição estocástica entre duas espécies é obtido das equações de Lotka-Volterra em presença do ruído branco,

$$
\left\{\begin{array}{l}
\dot{u}=u\left(a_{1}-b_{1} u-c_{1} v\right)+f(u, v) \xi_{u}(t) \\
\dot{v}=v\left(a_{2}-b_{2} u-c_{2} v\right)+g(u, v) \xi_{v}(t)
\end{array}\right.
$$

onde $u$ e $v$ representam as densidades das populações, $f(u, v) \xi_{u}(t)$ e $g(u, v) \xi_{v}(t)$ modelam a contribuição de forças aleatórias. $\xi_{u}(t)$ e $\xi_{v}(t)$ são os ruídos brancos Gaussianos independentes com média zero.

Palavras-chave: Competição estocástica, modelo Lotka-Volterra, modelo estocástico

\section{Introdução}

Muitas áreas interdisciplinares como Biologia Matemática, Bioestatística e Bioengenharia vêm crescendo rapidamente desde as últimas décadas. Em particular, muitas das aplicações biológicas são das áreas de dinâmica de populações e de epidemiologia. Modelar e analisar fenômenos biológicos requerem técnicas e ferramentas do Cálculo Estocástico e de várias outras disciplinas. Desde um ponto de vista aplicado, Cálculo Estocástico pode ser vagamente descrito como um ramo da Matemática que trata do cálculo infinitesimal para funções não diferenciáveis, e surgiu da necessidade de incluir aspectos não previsíveis no modelamento de vários fenômenos. $\mathrm{Na}$ área de Biologia, o Cálculo Estocástico é usado para modelar os efeitos, em uma ou várias populações, de variações estocásticas provenientes do processo de reprodução, migração, mudanças do meio ambiente, etc..

Neste trabalho apresentamos uma aplicação biológica: o modelo de competição estocástica de Lotka-Volterra entre duas espécies.

A competição entre duas espécies num sistema ecológico ha sido tradicionalmente formulado em relação a evolução no tempo com distribuição populacional uniforme no habitat através do modelo de Lotka-Volterra, [3], [6], [5]:

$$
\begin{gathered}
\left\{\begin{array}{l}
\dot{u}=u\left(a_{1}-b_{1} u-c_{1} v\right) \\
\dot{v}=v\left(a_{2}-b_{2} u-c_{2} v\right) .
\end{array}\right. \\
\left\{\begin{array}{l}
u(0)=u_{0} \geq 0 \\
v(0) \quad=v_{0} \geq 0
\end{array}\right.
\end{gathered}
$$


onde,

- $u$ e $v$ representam as densidades das populações;

- $a_{1}$ e $a_{2}$ são as taxas de crescimento intrínseco das densidades de populações $u$ e $v$;

- $b_{1}$ e $c_{2}$ são os coeficientes de competição intraespecíficos;

- $c_{1}$ e $b_{2}$ são os coeficientes de competição interespecíficos, isto é, medem o quanto uma espécie dificulta o crescimento da outra;

- $u_{o}$ e $v_{o}$ são constantes não negativas.

O sistema (3)-(4), dependendo das relações entre os coeficientes $a_{i}, b_{i}, c_{i}$ pode ter, se existirem, os seguintes pontos críticos hiperbólicos:

$$
P_{1}=(0,0), P_{2}=\left(\frac{a_{1}}{b_{1}}, 0\right), P_{3}=\left(0, \frac{a_{2}}{c_{2}}\right), P_{4}=\left(\frac{a_{1} c_{2}-a_{2} c_{1}}{\Delta}, \frac{a_{2} b_{1}-a_{1} b_{2}}{\Delta}\right)
$$

onde $\Delta=b_{1} c_{2}-b_{2} c_{1} \neq 0$.

Dependendo dos coeficientes $a_{i}, b_{i}, c_{i}$ temos 4 casos de (3)-(4): Sobrevivência da espécie $v$, Sobrevivência da espécie $u$, Coexistência estável e Coexistência instável, tal como mostram os seguintes retratos de fase:
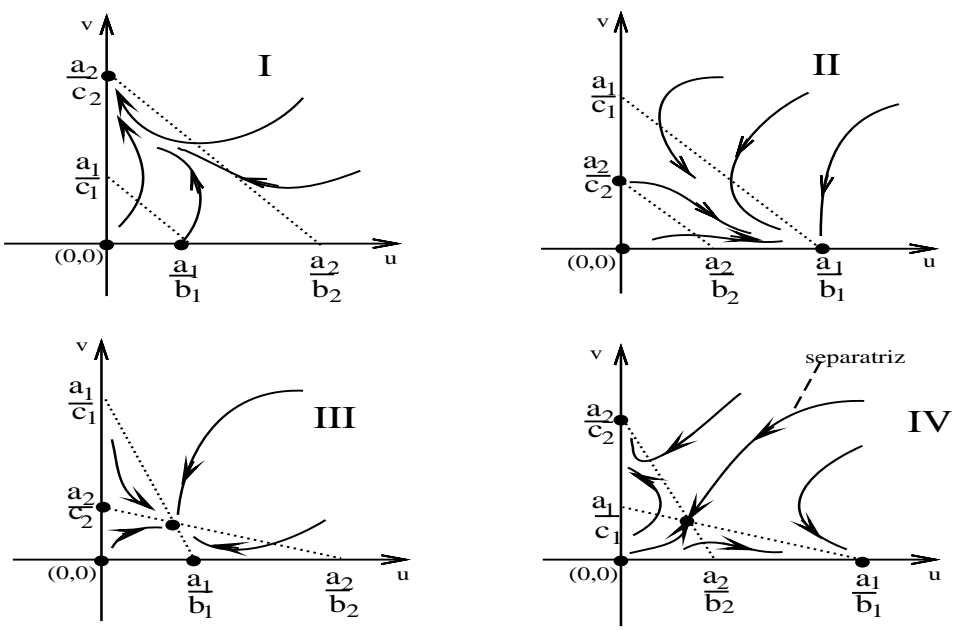

Figura 1: Retrato de fases de quatro possíveis casos do sistema (3)-(4): I- $\frac{a_{1}}{b_{1}}<\frac{a_{2}}{b_{2}}$, $\frac{a_{1}}{c_{1}}<$ $\frac{a_{2}}{c_{2}} \cdot P_{3}=\left(0, \frac{a_{2}}{c_{2}}\right)$ é atrator hiperbólico global para a região $u>0, v>0$. Neste caso temos sobrevivência da espécie $v$. II- $\frac{a_{1}}{b_{1}}>\frac{a_{2}}{b_{2}}, \frac{a_{1}}{c_{1}}>\frac{a_{2}}{c_{2}} . P_{2}=\left(\frac{a_{1}}{b_{1}}, 0\right)$ é atrator hiperbólico global para a região $u>0, v>0$. Neste caso temos sobrevivência da espécie $u$. III- $\frac{a_{1}}{b_{1}}<\frac{a_{2}}{b_{2}}, \frac{a_{1}}{c_{1}}>$ $\frac{a_{2}}{c_{2}} \cdot P_{4}=\left(\frac{a_{1} c_{2}-a_{2} c_{1}}{\Delta}, \frac{a_{2} b_{1}-a_{1} b_{2}}{\Delta}\right)$ é atrator hiperbólico global. Neste caso temos Coexistência estável das duas espécies. IV- $\frac{a_{1}}{b_{1}}>\frac{a_{2}}{b_{2}}, \frac{a_{1}}{c_{1}}<\frac{a_{2}}{c_{2}} \cdot P_{2}=\left(\frac{a_{1}}{b_{1}}, 0\right)$, é atrator, $P_{3}=\left(0, \frac{a_{2}}{c_{2}}\right)$ é atrator e $P_{4}$ é sela. Neste caso uma separatriz da sela $P_{4}$ separa o primeiro quadrante em duas regiões. 


\section{Resultados Matemáticos}

\section{Dedução do Modelo de Competição Estocástico}

Vamos introduzir um modelo de competição mais geral que possa relacionar o sistema (3)-(4) com o modelo de competição estocástica de Itô (2). Apesar que também existe o cálculo estocástico de Stratonovich, preferimos o cálculo de Itô para modelos biológicos, não somente por motivos computacionais, mas também porque a esperança matemática da solução de Itô coincide com a solução do caso determinístico, o que não acontece com a solução de Stratonovich [4].

Suponhamos que o novo sistema determinístico geral de interação de duas espécies satisfaz o sistema formado por duas equações diferenciais acopladas [2]:

$$
\left\{\begin{array}{l}
\dot{u}=u\left(g_{1}-d_{1}-\alpha_{21}\right)+\alpha_{12} v \\
\dot{v}=v\left(g_{2}-d_{2}-\alpha_{12}\right)+\alpha_{21} u
\end{array}\right.
$$

onde os parâmetros $g_{i}$ e $d_{i}$ são as taxas de crescimento e mortalidade para as populações $u$ e $v$, e $\alpha_{i j}$ é a taxa na qual a população $i(u$ ou $v$ ) é transformado na população $j(v$ ou u) ( por exemplo, quando há migração ou indivíduos infectados). Os parâmetros $g_{i}, d_{i}$ e $\alpha_{i j}$ podem ser funções da densidade da população das espécies $u$ e $v$ e do tempo $t$.

Vamos obter do sistema (5) o correspondente sistema estocástico (2).

De fato, sejam $X_{1}(t)$ e $X_{2}(t)$ variáveis aleatórias para o número das populações:

$$
X_{1}(t), X_{2}(t) \in[0, \infty), \quad t \in[0, \infty),
$$

e seja $X(t)=\left(X_{1}(t), X_{2}(t)\right)^{t}$ o vetor aleatório das duas populações. Para um $\Delta t$ suficientemente pequeno definimos a probabilidade de um simples nascimento ou morte na população $i$ por $g_{i} X_{i} \Delta t\left(X_{i} \rightarrow X_{i}+1\right)$ e $d_{i} X_{i} \Delta t\left(X_{i} \rightarrow X_{i}-1\right)$ respectivamente, $i=1,2$. A probabilidade que um indivíduo da população $i$ seja transformado em um indivíduo da população $j, i \neq j$, é $\alpha_{j i} X_{i} \Delta t\left(X_{i} \rightarrow X_{i}-1\right.$ e $\left.X_{j} \rightarrow X_{j}+1\right)$. Seja

$$
S\left(X_{1}, X_{2}\right)=\left(g_{1}+d_{1}+\alpha_{21}\right) X_{1}+\left(g_{2}+d_{2}+\alpha_{12}\right) X_{2} .
$$

Então a probabilidade de que não ocorram mudanças em ambas populações num intervalo muito pequeno $\Delta t$ é $1-S\left(X_{1}, X_{2}\right) \Delta t$.

Denotemos por $\Delta X_{i}(t)=X_{i}(t+\Delta t)-X_{i}(t), i=1,2$, então podemos escrever o incremento nas duas populações para um intervalo de tempo $\Delta t$ por $\Delta X=\left(\Delta X_{1}(t), \Delta X_{2}(t)\right)^{t}$. Para um $\Delta t$ muito pequeno calculamos a esperança matemática $\mathbb{E}(\Delta X)$ (ou média) e a matriz covariante de $\Delta X$.

$$
\begin{aligned}
\mathbb{E}(\Delta X) & \approx\left[(1,0)^{t} g_{1}+(-1,0)^{t} d_{1}+(-1,1)^{t} \alpha_{21}\right] X_{1} \Delta t \\
& +\left[(0,1)^{t} g_{2}+(0,-1)^{t} d_{2}+(1,-1)^{t} \alpha_{12}\right] X_{2} \Delta t \\
& +(0,0)^{t}\left[1-S\left(X_{1}, X_{2}\right) \Delta t\right] .
\end{aligned}
$$

Podemos escrever esta expressão em forma vetorial,

$$
\begin{aligned}
\mathbb{E}(\Delta X) & \approx\left(\begin{array}{l}
g_{1} X_{1}-d_{1} X_{1}-\alpha_{21} X_{1}+\alpha_{12} X_{2} \\
g_{2} X_{2}-d_{2} X_{2}-\alpha_{12} X_{2}+\alpha_{21} X_{1}
\end{array}\right) \Delta t \\
& =\mu \Delta t
\end{aligned}
$$

onde $\mu=\left(\mu_{1}, \mu_{2}\right)^{t}=\left(g_{1} X_{1}-d_{1} X_{1}-\alpha_{21} X_{1}+\alpha_{12} X_{2}, g_{2} X_{2}-d_{2} X_{2}-\alpha_{12} X_{2}+\alpha_{21} X_{1}\right)^{t}$.

A variância de $\Delta X_{i}$ satisfaz

$$
\sigma_{i}^{2}=\sigma_{i i}=\mathbb{E}\left(\left(\Delta X_{i}\right)^{2}\right)-\mu_{i}^{2}(\Delta t)^{2} .
$$


A covariância de $\Delta X_{1}$ e $\Delta X_{2}$ é $\sigma_{12}=\mathbb{E}\left(\Delta X_{1} \Delta X_{2}\right)-\mu_{1} \mu_{2}(\Delta t)^{2}$. Assim, a matriz de covariância para $\Delta X_{1}$ e $\Delta X_{2}$ é

$$
\operatorname{Cov}(\Delta X)=V=\mathbb{E}\left(\Delta X \Delta X^{t}\right)-\mathbb{E}(\Delta X)(\mathbb{E}(\Delta X))^{t} .
$$

Usando o fato que o produto $\mathbb{E}(\Delta X)(\mathbb{E}(\Delta X))^{t} \approx \mu \mu^{t}(\Delta t)^{2}$, é de ordem $(\Delta t)^{2}$,para $\Delta t$ pequeno, temos

$$
\begin{aligned}
V & \approx \mathbb{E}\left(\Delta X \Delta X^{t}\right) \\
& =\mathbb{E}\left(\begin{array}{cc}
\left(\Delta X_{1}\right)^{2} & \Delta X_{1} \Delta X_{2} \\
\Delta X_{2} \Delta X_{1} & \left(\Delta X_{2}\right)^{2}
\end{array}\right) \\
& \approx\left(\begin{array}{cc}
\sigma_{11} & \sigma_{12} \\
\sigma_{21} & \sigma_{22}
\end{array}\right) .
\end{aligned}
$$

Em particular,

$$
\begin{aligned}
\sigma_{i i} & =\mathbb{E}\left[\left(\Delta X_{i}\right)^{2}\right] \\
& \approx\left[(1)^{2} g_{i} X_{i}+(-1)^{2} d_{i} X_{i}+(-1)^{2} \alpha_{j i} X_{i}+(1)^{2} \alpha_{i j} X_{j}\right] \Delta t
\end{aligned}
$$

$\mathrm{e}$

$$
\sigma_{i j}=\mathbb{E}\left(\Delta X_{i} \Delta X_{j}\right) \approx-\left[\alpha_{21} X_{1}+\alpha_{12} X_{2}\right] \Delta t \quad \text { para } i, j=1,2 \text {, e } i \neq j .
$$

Portanto a matriz covariância $V \approx C \Delta t$, onde $C$ é dado por

$$
\left(\begin{array}{cc}
\left(g_{1}+d_{1}\right) X_{1}+\alpha_{21} X_{1}+\alpha_{12} X_{2} & -\alpha_{21} X_{1}-\alpha_{12} X_{2} \\
-\alpha_{21} X_{1}-\alpha_{12} X_{2} & \left(g_{2}+d_{2}\right) X_{2}+\alpha_{21} X_{1}+\alpha_{12} X_{2}
\end{array}\right)
$$

A matriz $C$ é positivamente definida [1], por isso $C$ tem uma raiz quadrada positiva dada por

$$
B=\sqrt{C} \approx \sqrt{V / \Delta t} .
$$

Quando $X(t)$ é suficientemente grande, podemos assumir que $\Delta X(t)$ tem uma distribuição normal com vetor média $\mu \Delta t$ e matriz covariância $B^{2} \Delta t=C \Delta t$ (isto é, $\Delta X(t) \sim N\left(\mu \Delta t, B^{2} \Delta t\right)$ ). Se $\xi=\left(\xi_{1}, \xi_{2}\right)^{t} \sim N(0, I)$ é um processo bidimensional de Wiener, então

$$
\mu \Delta t+B \sqrt{\Delta t} \xi \sim N\left(\mu \Delta t, B^{2} \Delta t\right)
$$

Assim, $X(t+\Delta t)$ pode ser escrito em forma vetorial:

$$
\begin{aligned}
X(t+\Delta t) & =X(t)+\Delta X(t) \\
& =X(t)+\mu \Delta t+B \sqrt{\Delta t} \xi .
\end{aligned}
$$

Expressando esta equação em termos de $X_{1}$ e $X_{2}$, denotando $B=\left(B_{i j}\right)$,

$$
\begin{aligned}
& X_{1}(t+\Delta t)=X_{1}(t)+\mu_{1} \Delta t+B_{11} \xi_{1} \sqrt{\Delta t}+B_{12} \xi_{2} \sqrt{\Delta t} \\
& X_{2}(t+\Delta t)=X_{2}(t)+\mu_{2} \Delta t+B_{21} \xi_{1} \sqrt{\Delta t}+B_{22} \xi_{2} \sqrt{\Delta t}
\end{aligned}
$$

Se $\Delta t \rightarrow 0$, e assumindo que a integral estocástica exista, então $\xi_{i} \sqrt{\Delta t} \rightarrow d W_{i}$, onde $W_{i}$ é um processo de Wiener. Então o sistema converge em média para o seguinte sistema de Itô

$$
\begin{aligned}
& d X_{1}(t)=\mu_{1} \Delta t+B_{11} d W_{1}(t)+B_{12} d W_{2}(t) \\
& d X_{2}(t)=\mu_{2} \Delta t+B_{21} d W_{1}(t)+B_{22} d W_{2}(t) .
\end{aligned}
$$

De novo, podemos escrever o sistema acima em forma vetorial

$$
d X=\mu d t+B d W
$$

onde

$$
\mu=\left(\begin{array}{l}
\mu_{1} \\
\mu_{2}
\end{array}\right)=\left(\begin{array}{l}
g_{1} X_{1}-d_{1} X_{1}-\alpha_{21} X_{1}+\alpha_{12} X_{2} \\
g_{2} X_{2}-d_{2} X_{2}-\alpha_{12} X_{2}+\alpha_{21} X_{1}
\end{array}\right)
$$

$B=\sqrt{C}$, e $W(t)=\left(W_{1}(t), W_{2}(t)\right)^{t}$. 


\section{Modelo de Competição Estocástico (2)}

Como no modelo (5), os parâmetros $g_{i}, d_{i}$ e $\alpha_{i j}$ são funções da densidade da população das espécies $u$ e $v$ e do tempo $t$, podemos escolher adequadamente estes coeficientes de interação da seguinte forma:

$g_{1}=a_{1}, d_{1}=b_{1} X_{1}+c_{1} X_{2}, g_{2}=a_{2}, d_{2}=b_{2} X_{1}+c_{2} X_{2}$, e $\alpha_{i j}=0$. Neste caso, o sistema de EDEs satisfaz a equação, $d X=\mu d t+B d W$, onde $\mu$ é a matriz coluna

$$
\mu=\left(\begin{array}{c}
X_{1}\left(a_{1}-b_{1} X_{1}-c_{1} X_{2}\right) \\
X_{2}\left(a_{2}-b_{2} X_{1}-c_{2} X_{2}\right)
\end{array}\right)
$$

e a matriz $B=\sqrt{C}$. A matriz $C$ dada por (6), é uma matriz diagonal, pois os $\alpha_{i j}=0$.

Assim, o sistema de EDEs de Itô para o modelo de competição de Lotka-Volterra baseado na escolha das taxas de crescimento e mortalidade tem a forma

$$
\begin{aligned}
& d X_{1}(t)=X_{1}\left(a_{1}-b_{1} X_{1}-c_{1} X_{2}\right) d t+\sqrt{X_{1}\left(a_{1}+b_{1} X_{1}+c_{1} X_{2}\right)} d W_{1} \\
& d X_{2}(t)=X_{2}\left(a_{2}-b_{2} X_{1}-c_{2} X_{2}\right) d t+\sqrt{X_{1}\left(a_{2}+b_{2} X_{1}+c_{2} X_{2}\right)} d W_{2},
\end{aligned}
$$

$X_{1}(t) \in[0, \infty)$ e $\quad X_{2}(t) \in[0, \infty)$.

Assim, podemos escrever esta equação na forma (2),

$$
\left\{\begin{array}{l}
d u=u\left(a_{1}-b_{1} u-c_{1} v\right) d t+f(u, v) d W_{1} \\
d v=v\left(a_{2}-b_{2} u-c_{2} v\right) d t+g(u, v) d W_{2}
\end{array}\right.
$$

onde $\xi_{u}=\frac{d W_{1}}{d t}$ e $\quad \xi_{v}=\frac{d W_{2}}{d t}$. Esta forma explícita de ruído branco representa a taxa de crescimento flutuante de cada espécie.

\section{Método de Euler}

Cabe mencionar que uma solução de um modelo estocástico a diferença de uma solução do sistema determinístico, é uma distribuição de probabilidade para cada uma das variáveis aleatórias, e um caminho de amostra ao longo do tempo ou espaço é uma realização desta distribuição.

Usamos o método de Euler [4] para aproximar a solução de (7):

$$
\left(u\left(t_{i}\right), v\left(t_{i}\right)\right)=\left(u_{i}, v_{i}\right),
$$

para $t_{i}=0, \Delta t, 2 \Delta t, \cdots, T, i=0,1, \cdots, k-1$, obtemos

$$
\begin{aligned}
& u_{i+1}=u_{i}+u_{i}\left(a_{1}-b_{1} u_{i}-c_{1} v_{i}\right) \Delta t+\sqrt{u_{i}\left(a_{1}+b_{1} u_{i}+c_{1} v_{i}\right)} \sqrt{\Delta t} \xi_{1 i} \\
& v_{i+1}=v_{i}+v_{i}\left(a_{2}-b_{2} u_{i}-c_{2} v_{i}\right) \Delta t+\sqrt{v_{i}\left(a_{2}+b_{2} u_{i}+c_{2} v_{i}\right)} \sqrt{\Delta t} \xi_{2 i},
\end{aligned}
$$

onde $\xi_{1 i}$ e $\xi_{2 i}$ são dois processos normais independentes de Wiener da forma $\sqrt{\Delta t} N(0,1)$, onde $N(0,1)$ a distribuição normal de uma variável aleatória com média zero e variância 1 , e $\Delta t=T / N$ para algum número positivo $N$.

A modo de exemplo, analisemos o seguinte sistema:

$$
\begin{gathered}
\left\{\begin{array}{c}
d X_{1}(t)=X_{1}\left(4-0,06 X_{1}-0,04 X_{2}\right) d t+\sqrt{X_{1}\left(4+0,06 X_{1}+0,04 X_{2}\right)} d W_{1} \\
d X_{2}(t)=X_{2}\left(3-0.02 X_{1}-0.08 X_{2}\right) d t+\sqrt{X_{1}\left(3+0.02 X_{1}+0.08 X_{2}\right)} d W_{2},
\end{array}\right. \\
\left\{\begin{array}{l}
X_{1}(0)=50 \\
X_{2}(0)=25
\end{array}\right.
\end{gathered}
$$


onde, $a_{1}=4, b_{1}=0,06, c_{1}=0,04, a_{2}=3, b_{2}=0,02, c_{2}=0,08$.

Um análise rápida permite-nos ver que o ponto $(50,25)$ é um ponto de equilíbrio estável no modelo determinístico do sistema (8).

A figura 2 mostra uma trajetória estocástica para as duas espécies em competição como função do tempo.

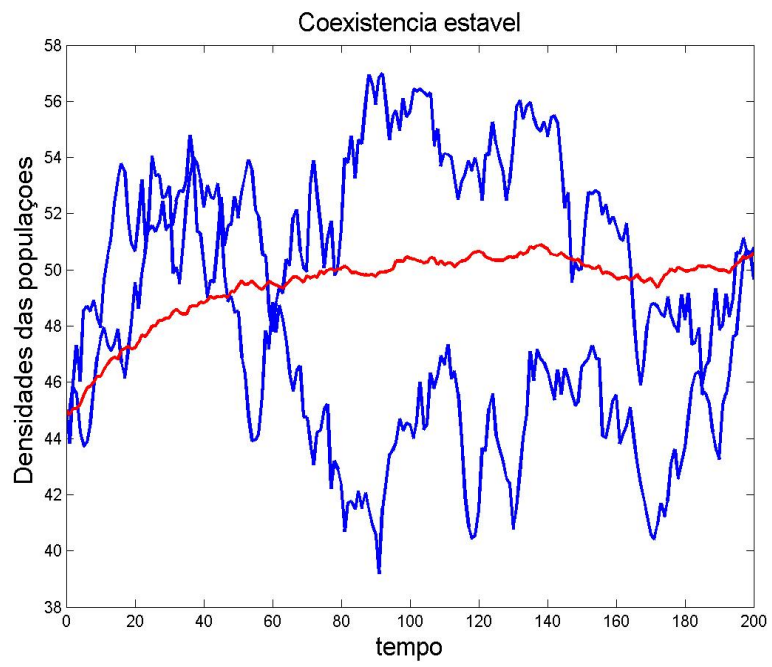

Figura 2: Uma simples trajetória do modelo de Lotka-Volterra como uma função do tempo com os seguintes valores dos parâmetros, $a_{1}=4, b_{1}=0,06, c_{1}=0.04, . a_{2}=3, b_{2}=0.02, c_{2}=$ $0.08, X_{1}(0)=50, X_{2}(0)=25, T=200, K=50000$ e $\sigma=0,8$.

Na figura 3 ilustramos um plano de fase com os valores de equilíbrio.

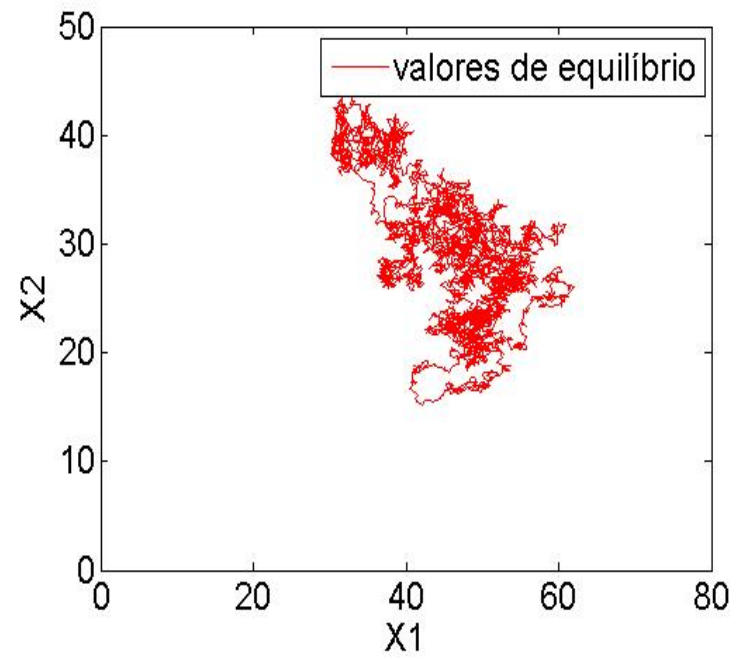

Figura 3: Região que indica os valores de equilíbrio do sistema (8) com condição inicial $X_{1}(0)=$ $50, X_{2}(0)=25$.

\section{Referências}

[1] A.G. Kurosh, Curso de Álgebra Superior (em Russo), Editora Nauka, Moscou 1971. 
[2] Linda J.S. Allen, An introduction to Stochastic process with Aplication to Biology. Pearson Education,INC, New Jersey, 2003.

[3] A.J. Lotka, Elements of Physical Biology. William and wilkins, Baltimore, 1925.

[4] Oksendal, B., Stochastic Differential Equation An Introduction with Aplications, SpringerVerlag, Berlin, New York, 2000.

[5] Villanueva Z. David, Sistema de Competição Lotka-Volterra, Dissertação de Mestrado, IME-USP, 2002.

[6] V. Volterra, Variazioni e Fluttuazioni del numero d'individui in specie d'animali conviventi, Mem. Acad. Lincei 2 (1926) 31-113. 